\title{
İkinci Mertebeden Gecikmeli Bir Diferansiyel Denklemin Hyers-Ulam Rassias Kararlılı̆̆
}

\author{
Hyers-Ulam Rassias Stability of a Second Order Delay Differential Equation
}

\section{Emel BİÇER*}

Bingöl Üniversitesi, Fen-Edebiyat Fakültesi, Matematik Bölümü, 12000, Bingöl

\begin{tabular}{|c|c|c|}
\hline - Geliş tarihi / Received: 09.11.2017 & - Düzeltilerek geliș tarihi / Received in revised form: 27.02.2018 & - Kabul tarihi / Accepted: 09.03.2018 \\
\hline
\end{tabular}

\section{Öz}

Bu çalışmada, ilk olarak $f$ bir sürekli fonksiyon ve $\tau$ negatif olmayan reel bir sabit olmak üzere $y^{\prime}(t)+f(t, y(t-\tau))=0$,

şeklindeki birinci mertebeden sabit gecikmeli bir diferansiyel denklemin Banach sabit nokta teoremi kullanılarak HyersUlam Rassias kararlılığı gösterildi. Buradan elde edilen sonuçtan faydalanılarak $f, h$ sürekli fonksiyonlar olmak üzere $y^{\prime \prime}(t)+f(t, y(t-\tau)) y^{\prime}(t-\tau)+h(t, y(t-\tau))=0$

biçimindeki ikinci mertebeden sabit gecikmeli bir diferansiyel denklemin Hyers-Ulam Rassias kararlılığı araştırıldı. Ayrıca çalışmadaki teorik analizleri açıklamak için bir örnek verildi.

Anahtar kelimeler: Banach Sabit Nokta Teoremi, Gecikmeli Diferansiyel Denklem, Hyers-Ulam Rassias Kararlılık

\begin{abstract}
In this study, firstly, we used Banach fixed point theorem to show that the Hyers-Ulam Rassias stability of a first order delay differential equation with constant delay of the form

$y^{\prime}(t)+f(t, y(t-\tau))=0$

where $f$ is continuous function and $\tau$ is a nonnegative real constant. By taking advantage of this result, we investigated Hyers-Ulam Rassias stability of a second order delay differential equation with constant delay of the form $y^{\prime \prime}(t)+f(t, y(t-\tau)) y^{\prime}(t-\tau)+h(t, y(t-\tau))=0$,

where $f, h$ are continuous functions and $\tau$ is a nonnegative real constant. Also we presented an example to illustrate the theoretical analysis.
\end{abstract}

Keywords: Banach Fixed Point Theorem, Delay Differential Equation, Hyers-Ulam Rassias Stability

*Emel BİÇER; ebicer@bingol.edu.tr; Tel: (0426) 21600 12; orcid.org/0000-0003-2085-2529 


\section{Giriş}

1940 yılında Wisconsin Üniversitesi'nde yapilan bir konferansta, Ulam'in ortaya attığ problemlerden bir tanesi şu şekildeydi:

$\left(G_{1}, \cdot\right)$ bir grup ve $\left(G_{2}, *\right), d(.,$.$) metriği ile bir$ metrik grup olsun. $\varepsilon>0$ sayisı verilsin. Her $x, y \in G_{1} \quad$ için $\quad d(h(x . y), h(x) * h(y))<\delta$ eşitsizliğini sağlayan $h: G_{1} \rightarrow G_{2}$ bir dönüşüm ise, her $\quad x \in G_{1}$ için $d(h(x), H(x))<\varepsilon$ ile $H: G_{1} \rightarrow G_{2}$ bir homomofizm olacak şekilde bir $\delta>0$ sayısı var mıdır? Diğer bir deyişle hangi şartlar altında bir yaklaşım homomorfizmine yaklaşan bir homomorfizm vardır? (Hyers, 1941).

Bu soru, Hyers (1941) tarafindan Banach uzayları için yanıtlanmıştır. Ulam'ın probleminin bir genelleştirmesi olarak fonksiyonel denklemlerin yerine diferansiyel denklemlerin kullanılmasiyla yeni bir çalışma alanı ortaya çıkmıştır. Böylece pek çok araştırmacı çeşitli diferansiyel denklemlerin Hyers-Ulam kararlılığ çalı̧̧malar yapmıştır.

$x^{\prime}(t)=\lambda x(t)$ şeklinde birinci mertebeden lineer diferansiyel denklemi ile ifade edilen bir otonom sistemi düşünelim. Eğer bu diferansiyel denklemin genel çözümü ve bir başlangıç koşulu biliniyorsa, bu sistemin geçmişi, şimdiki anı ve geleceği tamamen belirlenmiştir. Böylece, bu sistem "öngörülebilir"dir. Bazen, dıştan kaynaklanan bir sıkıntı nedeniyle, sistem $x^{\prime}(t)=\lambda x(t)$ diferansiyel denklemi ile belirlenemeyebilir. $\mathrm{Bu}$ durumda sistem $\left|x^{\prime}(t)-\lambda x(t)\right| \leq \varepsilon$ gibi bir eşitsizlikle ifade edilir. $\mathrm{Bu}$ durumda problemli sistemin geleceği tam olarak öngörülemez. Sistem, dışarıdan kaynaklı sıkıntılardan dolayı tamamen öngörülemez olmasına rağmen, eğer sistemin "reel" geleceği sınırlı bir hata ile $x^{\prime}(t)=\lambda x(t)$ diferansiyel denkleminin çözümünü takip ediyorsa, $x^{\prime}(t)=\lambda x(t)$ diferansiyel denklemi Hyers-Ulam kararlılığa sahiptir, denir. Eğer hata sınırı çok büyük ise denklem Hyers-Ulam kararlılığa sahip değildir, denir.

Alsina ve Ger (1998) birinci mertebeden lineer bir diferansiyel denklemin Hyers-Ulam kararlılığını araştıran ilk yazarlardır.

Son dönemde bir çok araştırmacı tarafından farklı yöntemler kullanılarak ikinci mertebeden diferansiyel denklemlerin Hyers-Ulam kararlıllı̆ araştırılmaktadır. 2009 yılında, Li ve Shen

$$
y^{\prime \prime}+p(x) y^{\prime}+q(x) y+r(x)=0
$$

biçimindeki ikinci mertebeden bir diferansiyel denklemin Hyers-Ulam kararlılığını araştırmıştır (Li ve Shen, 2009).

2013 yılında, Otrocol ve Ilea

$x^{\prime}(t)=f(t, x(t), x(g(t)))$

biçimindeki diferansiyel denklemin Hyers-Ulam kararlılığını araştırmıştır (Otrocol ve Ilea, 2013).

Tunç ve Biçer (2015), sabit nokta teorisinden faydalanarak birinci mertebeden gecikmeli bir diferansiyel denklemin Hyers-Ulam kararlılığını araştırmıştır. Ayrıca Biçer ve Tunç (2017), ikinci mertebeden kısmi türevli bir diferansiyel denklemin Hyers-Ulam kararlılığını araştırırken integral çarpanı metodundan faydalanmıştır.

Bu çalışmada, ilk olarak

$$
y^{\prime}(t)+f(t, y(t-\tau))=0
$$

şeklindeki birinci mertebeden sabit gecikmeli bir diferansiyel denklemin Hyers-Ulam Rassias kararlılığı gösterildi. Daha sonra $f, h$ sürekli fonksiyonlar ve $\tau$ negatif olmayan bir sabit olmak üzere

$$
y^{\prime \prime}(t)+f(t, y(t-\tau)) y^{\prime}(t-\tau)+h(t, y(t-\tau))=0
$$

biçimindeki ikinci mertebeden sabit gecikmeli bir diferansiyel denklemin Hyers-Ulam Rassias kararlılığı araştırıldı.

\section{2. Ön Bilgiler}

Tanım 2.1. $y:\left[t_{0}-\tau, T\right] \rightarrow \mathbb{R}$ fonksiyonunun verilen $\varepsilon \geq 0, \psi \in C\left[t_{0}-\tau, t_{0}\right]$ ve $T>t_{0}$, $t_{0}, T \in \mathbb{R}$ için

$$
\left\{\begin{array}{l}
\left|y^{\prime \prime}(t)+f(t, y(t-\tau)) y^{\prime}(t-\tau)+h(t, y(t-\tau))\right| \leq \varepsilon, \quad t \in\left[t_{0}, T\right] \\
|y(t)-\psi(t)| \leq \varepsilon, \quad t \in\left[t_{0}-\tau, t_{0}\right],
\end{array}\right.
$$

eşitsizliğini sağladığını varsayalım. Bu durumda, eğer 
$\left\{\begin{array}{l}y_{0}^{\prime \prime}(t)=-f\left(t, y_{0}(t-\tau)\right) y_{0}^{\prime}(t-\tau)-h\left(t, y_{0}(t-\tau)\right), \quad t \in\left[t_{0}, T\right] \\ y_{0}(t)=\psi(t), \quad t \in\left[t_{0}-\tau, t_{0}\right]\end{array}\right.$

denklemini ve

$\left|y(t)-y_{0}(t)\right| \leq K(\varepsilon)$

eşitsizliğini sağlayan bir $y_{0}:\left[t_{0}-\tau, T\right] \rightarrow \mathbb{R}$ fonksiyonu varsa (1) denklemine Hyers-Ulam kararlıdır, denir. Burada $K(\varepsilon), \lim _{\varepsilon \rightarrow 0} K(\varepsilon)=0$ koşulunu sağlayan $\varepsilon$ ' a bağlı bir ifadedir. $y$ ve $y_{0}{ }^{\prime}$ a bağlı olmayan $\phi, \varphi \in C\left[t_{0}-\tau, T\right]$ fonksiyonları için yukarıdaki tanımda $\varepsilon$ ve $K(\varepsilon)$ ifadelerinin yerine sirasiyla $\phi(t)$ ve $\varphi(t)$ fonksiyonları yazılırsa bu durumda (2) denklemi Hyers-Ulam Rassias kararlıdır (genelleştirilmiş Hyers-Ulam kararlıl1k), denir.

$\mathrm{Bu}$ çalışmada verilen denklemin Hyers-Ulam Rassias kararlılığını göstermek için aşağıdaki teoremden faydalanılacaktır.

Teorem 2.1. Banach Sabit Nokta Teoremi. $(X, d)$ bir tam metrik uzay ve $P: X \rightarrow X$ bir daralma dönüşümü olsun. Yani her $x, y \in X$ için

$d(P x, P y) \leq \alpha d(x, y)$

olacak şekilde $\alpha \in[0,1)$ mevcut olsun. $\mathrm{Bu}$ durumda $P a=a$ olacak şekilde bir tek $a \in X$ vardır. Ayrıca $a=\lim _{n \rightarrow \infty} P^{n} x$ olmak üzere her $x \in X$ için

$d(a, x) \leq \frac{1}{1-\alpha} d(x, P x)$

olur (Gordji vd., 2011).

\section{Hyers-Ulam Rassias Kararlılık}

$I=\left[t_{0}-\tau, T\right]$ kapalı aralığı verilsin. Aşağıdaki teoremde (1) denklemi ile verilen birinci mertebeden sabit gecikmeli bir diferansiyel denklemin Hyers-Ulam Rassias kararlı olduğu gösterilecektir. Buradan elde edilen verilerden faydalanılarak (2) denkleminin Hyers-Ulam Rassias kararlılığı araştırılacaktır.

Teorem 3.1. $I=\left[t_{0}-\tau, T\right]$ için $f: I \times \mathbb{R} \rightarrow \mathbb{R}$ sürekli fonksiyonu $k$ sabiti ile birinci değişkene göre Lipschitz şartını sağlasın. Yani $t \in I$ ve $x_{1}, x_{2} \in \mathbb{R}$ için

$\left|f\left(t, x_{1}(t)\right)-f\left(t, x_{2}(t)\right)\right| \leq k\left|x_{1}(t)-x_{2}(t)\right|$

eşitsizliği sağlansın. $0<k L<1, L \in(0,1)$ olmak üzere $k, L$ pozitif sabitler olsun. $\phi: I \rightarrow(0, \infty)$ sürekli fonksiyonu için

$\int_{t_{0}}^{t} \phi(s) d s \leq L \phi(t)$

eşitsizliği sağlansın. Eğer bir $\quad y: I \rightarrow \mathbb{R}$ sürekli fonksiyonu için

$\left\{\begin{array}{l}\left|y^{\prime}(t)+f(t, y(t-\tau))\right|<\phi(t), t \in\left[t_{0}, T\right] \\ y(t)-\psi(t) \mid \leq \phi(t), t \in\left[t_{0}-\tau, t_{0}\right]\end{array}\right.$

eşitsizliği sağlanırsa bu durumda her $t \in I$ için

$\left\{\begin{array}{l}y_{0}^{\prime}(t)=-f\left(t, y_{0}(t-\tau)\right), t \in\left[t_{0}, T\right] \\ y_{0}(t)=\psi(t), t \in\left[t_{0}-\tau, t_{0}\right],\end{array}\right.$

$\left|y(t)-y_{0}(t)\right| \leq \frac{L}{1-k L} \phi(t)$

olacak şekilde bir tek $y_{0}: I \rightarrow \mathbb{R}$ sürekli fonksiyonu vardır.

İspat. $C, I$ dan $\mathbb{R}$ ye tüm sürekli fonksiyonların uzayı olsun. $d$ metriği

$d(\zeta, \eta)=\sup _{t \in I} \frac{|\zeta(t)-\eta(t)|}{\phi(t)}$

ile verilsin. $C$ üzerinde $P$ operatörü her $\zeta \in C$ için

$$
\left\{\begin{array}{l}
(P \zeta)(t)=\psi(t), t \in\left[t_{0}-\tau, t_{0}\right], \\
(P \zeta)(t)=\psi\left(t_{0}\right)-\int_{t_{0}}^{t} f(s, \zeta(s-\tau)) d s, t \in\left[t_{0}, T\right]
\end{array}\right.
$$

şeklinde tanımlansın. (3) ve (4) den $t \in\left[t_{0}, T\right]$ için

$d(P \zeta, P \eta)=\sup _{t \in I} \frac{\left|\int_{t_{0}}^{t}[f(s, \zeta(s-\tau))-f(s, \eta(s-\tau))] d s\right|}{\varphi(t)}$ 


$$
\begin{aligned}
& \sup _{t \in I} \frac{\int_{t_{0}}^{t} k|\zeta(s)-\eta(s)| d s}{\phi(t)} \\
& \leq \sup _{t \in I} \frac{\int_{t_{0}}^{t} k \phi(s) \frac{|\zeta(s)-\eta(s)|}{\phi(s)} d s}{\phi(t)} \\
& \leq \sup _{t \in I} \frac{\int_{t_{0}}^{t} k \phi(s) \sup _{s \in I} \frac{|\zeta(s)-\eta(s)|}{\phi(s)} d s}{\phi(t)} \\
&=d(\zeta, \eta) \sup _{t \in I} \frac{\int_{t_{0}}^{t} k \phi(s) d s}{\phi(t)} \\
& \leq k L d(\zeta, \eta), \quad
\end{aligned}
$$

elde edilir. Ayrıca $t \in\left[t_{0}-\tau, t_{0}\right]$ için

$$
d(P \zeta, P \eta)=\psi(t)-\psi(t)=0,
$$

olduğundan

$$
d(P \zeta, P \eta) \leq k L d(\zeta, \eta)
$$

eşitsizliği yazılabilir. Bu durumda Teorem 2.1 den $P y_{0}=y_{0}$ olacak şekilde bir tek $y_{0} \in C$ vardır. Ayrica $y_{0}$,

$$
\left\{\begin{array}{l}
y_{0}^{\prime}(t)=-f\left(t, y_{0}(t-\tau)\right), t \in\left[t_{0}, T\right] \\
y_{0}(t)=\psi(t), t \in\left[t_{0}-\tau, t_{0}\right]
\end{array}\right.
$$

eşitliğini sağlar. $\mathrm{O}$ halde Theorem 2.1 den her $y \in C$ için

$$
d\left(y_{0}, y\right) \leq \frac{1}{1-k L} d(y, P y)
$$

eşitsizliği yazılabilir. Ayrıca her $t \in\left[t_{0}, T\right]$ için (5) eşitsizliğinden $\forall t \in\left[t_{0}, T\right]$ için

$$
-\phi(t) \leq y^{\prime}(t)+f(t, y(t-\tau)) \leq \phi(t),
$$

elde edilir. Eğer yukarıdaki eşitsizlikte her bir terim için $t_{0}$ dan $t$ ye integral alınırsa,

$$
\begin{array}{r}
\left|y(t)-\psi\left(t_{0}\right)+\int_{t_{0}}^{t} f(s, y(s-\tau)) d s\right| \leq \int_{t_{0}}^{t} \phi(s) d s \\
\leq L \phi(t), \quad t \in\left[t_{0}, T\right]
\end{array}
$$

olur. Bu eşitsizlik

$$
\frac{|y(t)-(P y)(t)|}{\phi(t)} \leq L
$$

şeklinde ifade edilirse,

$$
\sup _{t \in I} \frac{|y(t)-(P y)(t)|}{\phi(t)} \leq L
$$

yazılabilir. Yani

$$
d(y, P y) \leq L
$$

olur. O halde (6) ve (7) den $t \in I$ için

$$
\left|y(t)-y_{0}(t)\right| \leq \frac{L}{1-k L} \phi(t)
$$

elde edilir. Böylece ispat tamamlanır.

Şimdi de Teorem 3.1 den faydalanılarak (2) denklemi ile belirtilen ikinci mertebeden sabit gecikmeli bir diferansiyel denklemin Hyers-Ulam Rassias kararlılığı araştırılacaktır.

Teorem 3.2. $I=\left[t_{0}-\tau, T\right]$ olmak üzere $K: I \times \mathbb{R}$ $\rightarrow \mathbb{R}$ sürekli fonksiyonu her $t \in I$ ve $x_{1}, x_{2} \in \mathbb{R}$ için aşağıdaki şartı sağlasın.

$\left|K\left(t, x_{1}(t)\right)-K\left(t, x_{2}(t)\right)\right| \leq k\left|x_{1}(t)-x_{2}(t)\right|$.

Burada

$$
\begin{aligned}
& K(t, y(t-\tau))=-f(t, y(t-\tau)) y(t)- \\
& \int_{t_{0}}^{t} \frac{\partial}{\partial s}[f(s, y(s-\tau))] y(s) d s+ \\
& \int_{t_{0}}^{t} h(s, y(s-\tau)) d s-r\left(t_{0}\right)
\end{aligned}
$$

şeklindedir. $\phi: I \rightarrow(0, \infty)$ sürekli fonksiyonu için Teorem 3.1 deki şartlar sağlansın. Eğer bir $y: I \rightarrow \mathbb{R}$ sürekli fonksiyonu için 
$\left\{\left|y^{\prime \prime}(t)+f(t, y(t-\tau)) y^{\prime}(t-\tau)+h(t, y(t-\tau))\right|<\varphi(t), \quad t \in\left[t_{0}, T\right]\right.$

||$y(t)-\psi(t) \mid \leq \varphi(t), \quad t \in\left[t_{0}-\tau, t_{0}\right]$

eşitsizliği sağlanırsa bu durumda her $t \in I$ için

$\left\{\begin{array}{l}y_{0}^{\prime \prime}(t)=-f\left(t, y_{0}(t-\tau)\right) y_{0}^{\prime}(t-\tau)-h\left(t, y_{0}(t-\tau)\right), \quad t \in\left[t_{0}, T\right] \\ y_{0}(t)=\psi(t), \quad t \in\left[t_{0}-\tau, t_{0}\right]\end{array}\right.$

ve

$\left|y(t)-y_{0}(t)\right| \leq \frac{L}{1-k L} \phi(t)$

olacak şekilde bir tek $y_{0}: I \rightarrow \mathbb{R}$ sürekli fonksiyonu vardir.

İspat. (8) deki

$\left|y^{\prime \prime}(t)+f(t, y(t-\tau)) y^{\prime}(t-\tau)+h(t, y(t-\tau))\right| \leq \varphi(t)$.

eşitsizliğinden

$-\varphi(t) \leq y^{\prime \prime}(t)+f(t, y(t-\tau)) y^{\prime}(t-\tau)+h(t, y(t-\tau)) \leq \varphi(t)$

yazılır. $\mathrm{Bu}$ eşitsizlikte, $t_{0}$ dan $t$ ye terim terim integral alınırsa,

$\left|y^{\prime}(t)-y^{\prime}\left(t_{0}\right)+\int_{t_{0}}^{t} f(s, y(s-\tau)) y^{\prime}(t-\tau) d s+\int_{t_{0}}^{t} h(s, y(s-\tau)) d s\right| \leq \int_{t_{0}}^{t} \varphi(s) d s$

elde edilir. Kısmi integrasyon işlemi ile düzenleme yapılırsa

$\left|\begin{array}{l}y^{\prime}(t)+f(t, y(t-\tau)) y(t-\tau)- \\ \int_{t_{0}}^{t} \frac{\partial}{\partial s}[f(s, y(s-\tau))] y(s-\tau) d s+\int_{t_{0}}^{t} h\left(s, y(s-\tau) d s+r\left(t_{0}\right)\right.\end{array}\right| \leq \int_{t_{0}}^{t} \varphi(s) d s$ olur. Burada

$r\left(t_{0}\right)=f\left(t_{0}, y\left(t_{0}-\tau\right)\right) y\left(t_{0}-\tau\right)+y^{\prime}\left(t_{0}\right)$

şeklindedir.

$K(t, y(t-\tau))=-f(t, y(t-\tau)) y(t)+$

$\int_{t_{0}}^{t} \frac{\partial}{\partial s}[f(s, y(s-\tau))] y(s) d s-$

$\int_{t_{0}}^{t} h(s, y(s-\tau)) d s-r\left(t_{0}\right)$

fonksiyonunun yukarıdaki eşitsizlikte yerine yazılmasıyla

$\left|y^{\prime}(t)-K(t, y(t-\tau))\right| \leq \int_{t_{0}}^{t} \phi(s) d s$

$\leq L \phi(t) \leq \phi(t)$ elde edilir. Böylece ispat tamamlanır.

Örnek. $\varepsilon<2 \tau$ pozitif bir sayı olmak üzere $I=[-\tau, 2 \tau-\varepsilon]$ kapalı aralığ verilsin. $y: I \rightarrow$ $\mathbb{R}$ sürekli fonksiyonu her $t \in I$ için

$\left|y^{\prime \prime}(t)+f(t, y(t-\tau)) y^{\prime}(t-\tau)+h(t, y(t-\tau))\right| \leq t^{2}+\varepsilon$

eşitsizliğini sağlasın. Bu durumda her $t \in I$ için

$L \phi(t)-\frac{1}{3} t^{3}-t \varepsilon \geq 0$,

olduğundan her bir $t \in I$ için

$\int_{t_{0}}^{t} \phi(s) d s=\int_{0}^{t}\left(s^{2}+\varepsilon\right) d s \leq \frac{1}{3} t^{3}+t \varepsilon \leq L \phi(t)$

yazılabilir. Bu durumda Teorem 3.2 den herhangi bir $t \in I$ için

$\left\{y_{0}^{\prime \prime}(t)=f\left(t, y_{0}(t-\tau)\right) y_{0}^{\prime}(t-\tau)+h(t, y(t-\tau)), \quad t \in[0,2 \tau-\varepsilon]\right.$

$\left\{y_{0}(t)=\psi(t), \quad t \in[-\tau, 0]\right.$,

ve

$\left|y(t)-y_{0}(t)\right| \leq \frac{L}{1-k L}\left(t^{2}+\varepsilon\right)$

olacak şekilde bir tek $y_{0}: I \rightarrow \mathbb{R}$ sürekli fonksiyonu vardır. Böylece (9) denkleminin Hyers-Ulam Rassias kararlı olduğu görülür.

\section{Sonuç}

$\mathrm{Bu}$ çalışmada ikinci mertebeden gecikmeli bir diferansiyel denklemin Hyers-Ulam ve HyersUlam Rassias kararlılığ 1 araştırılmıştır. İlgili denklemin kararlılığını göstermek için Banach daralma dönüşümü prensibinden faydalanılmıştır. İkinci mertebeden gecikmeli bir diferansiyel denklemin Hyers-Ulam kararlılığını araştırmak oldukça zor iken sabit nokta teorisinden faydalanıldığında bu zorlukların ortadan kalktığı görülmüştür.

\section{Kaynaklar}

Alsina, C. ve Ger, R., 1998. On some Inequalities and Stability Results Related to the Exponential Function. J. Inequal. Appl, 4, 373-380. 
Biçer, E. ve Tunç, C., 2017. On the Hyers-Ulam stability of certain partial differential equations of second order. Nonlinear Dyn. Syst. Theory. 17, 150-157.

Cimpean, DS. ve Popa, D., 2011. Hyers-Ulam stability of Euler's equation. Appl. Math. Lett, 9, 1539-1543.

Gordji, M. ve Cho, YJ., Ghaemi, MB. ve Alizadeh, B., 2011. Stability of the second order partial differential equations. J. Inequal. Appl, 81, 10 pp.

Huimin, L. ve Xiangkui, Z., 2013. Hyers-UlamRassias stability of second order partial differential equations. Ann. Differential Equations, 29, 430-437.

Hyers, Donald H., 1941. On the Stability of the Linear Functional Equation. Proc. Nat. Acad. Sci, U.S.A. 27, 222-224.

Jung, S.M. ve Brzdęk, J., 2010. Hyers-Ulam Stability of the Delay Equation $y^{\prime}(t)=\lambda y(t-\tau)$, Abstr. Appl. Anal, 110.

Jung, S.M., 2005. Hyers Ulam stability of linear differential equations of first order (III). J. Math. Anal. Appl, 311, 139-146.

Jung, S.M., 2006. Hyers Ulam stability of linear differential equations of first order (II). Appl. Math. Lett, 19, 854-858.

Jung, S.M., 2007. Hyers Ulam stability of first order linear partial differential equations with constant coefficients. Math. Inequal. Appl, 10, 261-266.

Jung, S.M., 2009. Hyers Ulam stability of linear partial differential equations of first order. Appl. Math. Lett, 22, 70-74.

Li, Y. and Shen, Y. (2009). Hyers-ulam stability of nonhomogeneous linear differential equations of second order. International Journal of Mathematics and Mathematical Analysis, 1-7.

Lungu, N. ve Popa, D., 2012. Hyers-Ulam stability of a first order partial differential equation. J.Math.Anal.Appl, 86-91.

Lungu, N. ve Popa, D., 2014. Hyers-Ulam stability of some partial differential equations. Carpathian J. Math, 30, 327334.

Otrocol, D. ve Ilea, V., 2013. Ulam Stability for a Delay Differential Equation. Cent. Eur. J. Math, 7, 1296-1303.

Rassias, TM., 1978. On the Stability of the Linear Mapping in Banach Spaces. Proc Amer Math. Soc, 72, 297-300.

Tunc, C. ve Bicer, E., 2015. Hyers-Ulam-Rassias stability for a first order functional differential equation. J. Math. Fundam. Sci, 47, 143-153.

Ulam, S. M., 1964. Problems in Modern Mathematics. Science Editions John Wiley \& Sons, Inc., New York. 